\title{
Life after Addiction-Post-Operative Pain Management in an Obstetrical Patient on Long-Term Buprenorphine Therapy
}

\author{
Kelly R Bowen, Jonathan D Baum \\ Department of Obstetrics and Gynecology, Jersey Shore University Medical Center, Neptune, USA \\ Email: kelly.bowen@hackensackmeridian.org, jonathan.baum@hackensackmeridian.org
}

How to cite this paper: Bowen, K.R. and Baum, J.D. (2017) Life after Addiction-Post-Operative Pain Management in an Obstetrical Patient on Long-Term Buprenorphine Therapy. Open Journal of Obstetrics and Gynecology, 7, 951-956. https://doi.org/10.4236/ojog.2017.79096

Received: August 12, 2017

Accepted: September 12, 2017

Published: September 15, 2017

Copyright (c) 2017 by authors and Scientific Research Publishing Inc. This work is licensed under the Creative Commons Attribution International License (CC BY 4.0).

http://creativecommons.org/licenses/by/4.0/

\begin{abstract}
A G6P2032 female, prior cesarean x3 with history of opioid addiction maintained on buprenorphine presented for scheduled repeat cesarean section. Pre-operatively, her maintenance dose of medication was held secondary to concerns for partial agonist effect. Post-operative pain control was suboptimal with the patient ultimately proceeding to withdrawal. Doses of hydromorphone were titrated to $10 \mathrm{mg}$ every 3 hours to avoid further withdrawal. Review of expert opinion after discharge recommended against holding buprenorphine therapy in the post-operative period. Pain management options include maintenance therapy with additional doses of opioid and non-opioid pain relieving medications.
\end{abstract}

\section{Keywords}

Buprenorphine, Methadone, Addiction, Pregnancy, Opiate Use in Pregnancy

\section{Introduction}

Rates of opiate addiction and subsequent treatment with long-term maintenance therapy are increasing nationwide. Heroin use in reproductive-aged females has increased $100 \%$ in the last 14 years [1]. Misuse of prescription opiates has also been increasing exponentially over the last decade. Between 1999 and 2010, nearly 48,000 American women died of prescription opiate overdose, a $400 \%$ increase [2]. Among pregnant women, use of prescription opioids has increased more than four-fold in the last 15 years, and the incidence of neonatal abstinence syndrome (NAS) has nearly tripled [3]. With more than $1 / 3$ of reproductive-aged females on Medicaid filling a prescription for opiates each year and the concomitant rise in the purity of heroin available in the United States, the stakes 
are high to assure timely care for these patients [4] [5]. The epidemic of opiate abuse has grown to such proportions that some communities, including New Jersey, have made naloxone (Narcan) available in all first response vehicles and even legal for non-prescribed possession by lay persons [6]. Medication-assisted treatment (MAT), also known as opioid maintenance therapy, has been the standard approach in opioid addiction treatment. Appropriate obstetrical care for patients with substance use habits includes early screening, open communication, and early transition to a long-term maintenance medication when appropriate. Methadone is a long-acting full opioid agonist that is orally effective and can be given once daily. In obstetrical patients, maintenance on methadone is considered the standard of care. However, recent evidence suggests maintenance on buprenorphine in pregnancy may decrease the severity of NAS [7]. Buprenorphine maintenance presents a unique challenge in pain treatment secondary to the high affinity of buprenorphine for the mu opioid receptor, presenting competition for binding sites with opiate painkillers [8]. In addition, opiate non-naive patients develop cross-tolerance, and require higher doses of analgesia at shorter intervals to achieve the same effect [8]. Pain is anticipated during the peripartum period and should be addressed in opiate-addicted patients; flexibility in dosage and frequency of medication administration is necessary. Given the cesarean delivery rate of up to $39 \%$, the need to manage post-operative pain following cesarean is becoming more common. With permission, we present a case of an opiate-addicted patient maintained on buprenorphine therapy and the challenges encountered in achieving adequate pain management in her post-operative course at a small community hospital in New Jersey.

\section{Case Report}

A 31 years old white female, gravida 6, para 3 underwent repeat cesarean section at 39 weeks gestation. Her prenatal course was significant for history of opioid addiction and prior cesarean section. Prior to pregnancy she was maintained on buprenorphine $12 \mathrm{mg}$ daily (8 $\mathrm{mg} \mathrm{AM}, 4 \mathrm{mg}$ PM) with dose remaining unchanged in pregnancy. In anticipation of the need for higher opiate doses to control post-operative pain, her buprenorphine was held 12 hours prior to surgery due to its partial agonist effect and potential for respiratory depression. The patient noted early withdrawal symptoms including GI upset, rhinorrhea, piloerection, diaphoresis and anxiety 18 hours after her last buprenorphine dose. Vital signs remained within normal limits and preoperative laboratory studies were unremarkable. Surgery began within 24 hours of her last buprenorphine dose. She received spinal blockade with bupivacaine $0.75 \%$ pre-operatively and the procedure was uncomplicated-she was delivered a $2.95 \mathrm{~kg}$ female infant with Apgar scores of 9 and 9 at one and five minutes respectively. The infant went to the newborn nursery for routine neonatal care. Her immediate post-operative pain was adequately controlled with $2 \mathrm{mg}$ morphine IVP followed by hydro- 
morphone $1 \mathrm{mg}$ IVP. To avoid dependence on IV opiates, a plan was made to start oral hydromorphone $2 \mathrm{mg}$ every 4 hours as needed for pain. Approximately 30 hours after her last buprenorphine dose, however, withdrawal symptoms worsened and her pain was not controlled.

Buprenorphine was held initially secondary to recommendations given as an outpatient pre-operatively by the patient's addiction-medicine specialist, based both on concerns over partial agonist effect and the possibility of respiratory suppression with concurrent high doses of opiate pain medication. By post-operative day \#2 the patient began to report worsening symptoms of withdrawal and continued to report pain scores in the 7-10/10 range despite receiving $16 \mathrm{mg}$ of hydromorphone $\mathrm{PO}$ in an 8 hour period in addition to ketorolac 30 $\mathrm{mg}$ IVP and alprazolam $6 \mathrm{mg}$. Vital signs showed elevation in blood pressure and heart rate, and the patient became persistently diaphoretic with rhinorrhea. Pain management and withdrawal reversal options were reviewed. Using a conversion table, $0.4 \mathrm{mg}$ of buprenorphine IV or IM was found to be equivalent to $7.5 \mathrm{mg}$ of hydromorphone PO. Assuming approximately equivalent bioavailability of IV/IM buprenorphine with buprenorphine PO, the patient's equianalgesic dose of hydromorphone $\mathrm{PO}$ to daily buprenorphine dose was calculated at 225 $\mathrm{mg}$ of hydromorphone for a 24 hour period, equaling approximately $9.4 \mathrm{mg}$ hydromorphone hourly [9]. However, secondary to improvement in pain with NSAIDs and concerns for respiratory suppression at high hydromorphone doses, the patient was transitioned to hydromorphone $10 \mathrm{mg}$ q3hrs as needed for pain with ibuprofen $800 \mathrm{mg}$ q8hrs. Self-rated pain scores improved and she was discharged to home on this dose on post-operative day \#3. The newborn began showing signs of withdrawal on day of life one and was transferred to the neonatal intensive care unit (NICU) for NAS; the infant was later discharged after weaning without incident. Buprenorphine therapy was resumed weeks later without incident by her addiction medicine specialist.

\section{Discussion}

Opiate addiction and subsequent treatment with maintenance therapy has been seen with increasing frequency nationally [1]. As the rates of addiction and subsequent recovery increase in reproductive-age females, obstetricians can expect to encounter increasing numbers of patients who will require screening and treatment, with particular attention to pain management becoming a necessity. While methadone has long been the standard maintenance medication for opiate addiction in pregnancy, use of buprenorphine has increased [7]. The increasing frequency with which buprenorphine has been chosen as a maintenance medication is likely due to a combination of factors, including patients' ease of use in the form of tablets and films that can be prescribed with several doses at a time rather than a single dose dispensed, and increased access through private offices rather than through addiction centers [7]. In addition, buprenorphine acts as a partial mu-opioid agonist and kappa-antagonist with lower intrinsic affinity 
than methadone against the mu-opioid receptor, thus reducing peak effect and creating a plateau-this makes the risk for overdose death lower with buprenorphine than methadone [7]. Buprenorphine has a longer duration of action than does methadone, making it an attractive choice for patients requiring several doses daily [7]. With the MOTHER study by Jones et al. demonstrating potential reductions in the severity of NAS in infants born to mothers maintained on buprenorphine, a strong possibility exists that women already maintained on buprenorphine prior to pregnancy will continue to remain on the drug during pregnancy rather than being converted to methadone [7] [10].

The MOTHER study additionally found that, while post-operative pain management presented a challenge in patients maintained on buprenorphine, the rates of effective pain management were not significantly different to those of patients maintained on methadone [7]. Multiple pain management regimens exist for opiate non-naive patients, but it is generally accepted that the patient ought to continue their maintenance medication through the day of surgery, or given an equivalent loading dose of either narcotic prior to or during surgery [11]. Patients on buprenorphine may be restarted on buprenorphine alone for management of post-operative pain, with addition of morphine or methadone recommended for additional pain control as needed [11]. If the duration of post-operative pain is likely to be prolonged, buprenorphine may be discontinued. Opioid analgesics or methadone may be initiated instead, with or without conversion back to buprenorphine upon discharge [8]. With the exception of mixed agonist-antagonist opioids including nalbuphine, butorphanol, and pentazocine, which may precipitate withdrawal, all other opiate-based medications are acceptable choices for breakthrough pain in patients maintained on buprenorphine [11].

Additionally it is important to recall that opiate non-naïve patients experience down-regulation of opioid receptors; this increases the need for intra- and post-operative analgesic doses by $30 \%-100 \%$ [11]. Intravenous PCA pump with elevated lockout intervals and close clinical monitoring can be considered, in addition to intra- or early post-operative equianalgesic administration of missed buprenorphine doses.

Early and consistent use of non-opiate analgesics (NSAIDs, acetaminophen) often proves helpful in reducing overall opiate consumption by decreasing post-operative inflammation [11]. Use of these medications either intra-operatively or immediately post-operatively in PACU prior to initial sensation of early post-operative pain (i.e. resolution of spinal blockade) will likely produce substantial reduction in post-operative pain.

Finally, early assessment of the patient's equianalgesic dosing is crucial to appropriate pain management in opiate non-naive patients [11]. While doses of opiates were titrated up quickly in this patient, her total equianalgesic dose, when appropriately calculated, was significantly greater than the amount of medication administered, and improvement in subjective pain scores was achieved 
when hydromorphone doses were appropriately increased. Prejudice against opiate non-naive patients can present a barrier in adequate pain management, with clinician fear of manipulation, concern for relapse, and concern for excessive sedation all being common reasons clinicians give for overly conservative pain management in these patients [8]. Inadequate treatment of acute pain may lead to decreased responsiveness to opioid analgesics, perpetuating pain management difficulties [8]. When faced with patients that have known opiate use or abuse history, planning for post-operative pain management including calculating equianalgesic doses, reviewing methods of post-operative pain management (i.e. use of PCA versus oral versus parenteral administration of medication), assessing medications available for use (including short- and long-acting agents) and discussion involving the patient directly may help to alleviate anxiety and set expectations for both clinician and patient [8].

Opiate use disorder in obstetrical patients presents a unique challenge where the needs of the patient must be balanced against the best interest of her fetus. Expert consensus opinion is that initiation or continuation on maintenance therapies (methadone, buprenorphine) is superior to attempts at abrupt discontinuation of the opiate of choice, and certainly superior to continued use [3] [7]. Post-operative pain management in the opiate non-naive patient involves careful planning of maintenance medication dosing, frequent assessment of self-reported pain scores and objective signs of excessive sedation on the part of clinical staff, and frequent, often-high doses of pain medication, with doses frequently rising above the level many clinicians are comfortable with. Buprenorphine, a relatively new maintenance medication in obstetrical patients, is being used with increased frequency in this population, and knowledge of its mechanisms of action and strategies for post-operative pain management is becoming necessary to care for these patients. Careful planning of the post-operative course can significantly reduce levels of patient discomfort and will help avoid withdrawal symptoms.

\section{References}

[1] CDC Factsheet (2015) Vital Signs-Today's Heroin Epidemic. Centers for Disease Control and Prevention, Atlanta.

[2] CDC Factsheet (2013) Vital Signs-Prescription Painkiller Overdoses; A Growing Epidemic, Especially among Women. Centers for Disease Control and Prevention, Atlanta.

[3] Jones, H., Deppen, K., Hudak, M., Leffert, L., McClelland, C., Sahin, L., et al. (2014) Clinical Care for Opioid-Using Pregnant and Postpartum Women: The Role of Obstetric Providers. AJOG, 210, 302-310. https://doi.org/10.1016/j.ajog.2013.10.010

[4] CDC Press Release (2015) Opioid Painkillers Widely Prescribed among Reproductive Age Women. Centers for Disease Control and Prevention, Atlanta.

[5] Peskoe, A. (2014) DEA Warns of High Purity Heroin in N.J. http://www.nj.com/monmouth/index.ssf/2014/09/dea warns of high purity heroi n in nj.html

[6] Davis, M. (2015) Amendment to Overdose Protection Act Protects First Responders 
Who Administer Narcan.

http://www.nj.com/mercer/index.ssf/2015/02/amendment to overdose prevention act protects firs.html

[7] Jones, H., Heil, S., Baewert, A., Arria, A., Kaltenbach, K., Martin, P., et al. (2012) Buprenorphine Treatment of Opioid-Dependent Pregnant Women: A Comprehensive Review. Addiction, 107, 5-27. https://doi.org/10.1111/j.1360-0443.2012.04035.x

[8] Alford, D., Compton, P. and Samet, J. (2006) Acute Pain Management for Patients Receiving Maintenance Methadone or Buprenorphine Therapy. Annals of Internal Medicine, 144, 127-134. https://doi.org/10.7326/0003-4819-144-2-200601170-00010

[9] Brennan, M., Fudin, J. and Perkin, R. (2016) Opioid Calculator. Practical Pain Management. http://opioidcalculator.practicalpainmanagement.com

[10] Jones, H., Fischer, G., Heil, S., Kaltenbach, K., Martin, P., Coyle, M., et al. (2012) Maternal Opioid Treatment: Human Experimental Research (MOTHER) -Approach, Issues and Lessons Learned. Addiction, 107, 28-35. https://doi.org/10.1111/j.1360-0443.2012.04036.x

[11] Mitra, S. and Sinatra, R. (2004) Perioperative Management of Acute Pain in the Opioid-Dependent Patient. Anesthesiology, 101, 212-227. https://doi.org/10.1097/00000542-200407000-00032

\section{Submit or recommend next manuscript to SCIRP and we will provide best service for you:}

Accepting pre-submission inquiries through Email, Facebook, LinkedIn, Twitter, etc. A wide selection of journals (inclusive of 9 subjects, more than 200 journals)

Providing 24-hour high-quality service

User-friendly online submission system

Fair and swift peer-review system

Efficient typesetting and proofreading procedure

Display of the result of downloads and visits, as well as the number of cited articles

Maximum dissemination of your research work

Submit your manuscript at: http://papersubmission.scirp.org/

Or contact ojog@scirp.org 